\title{
Effect of miR-192-5p on Osteogenic Differentiation of Bone Marrow Mesenchymal Stem Cells in Idiopathic Scoliosis by Regulating Wnt/B-catenin Signaling Pathway via RSPO1
}

\author{
Yifan Yang \\ first affiliated hospital of kunming medical university \\ Jing Xu \\ first affiliated hospital of kunming medical university \\ Qingxin Su \\ first affiliated hospital of kunming medical university \\ Yiran Wu \\ first affiliated hospital of kunming medical university \\ Qizheng Li \\ first affiliated hospital of kunming medical university \\ zongren Ma \\ first affiliated hospital of kunming medical university \\ tao ding ( $\nabla$ dingta01180@163.com) \\ first affiliated hospital of kunming medical university
}

\section{Research article}

Keywords: Idiopathic scoliosis, miR-192-5p, RSP01, Wnt/ $\beta$-catenin signaling pathway, Bone marrow mesenchymal stem cells, Osteogenic differentiation

Posted Date: November 1st, 2021

DOI: https://doi.org/10.21203/rs.3.rs-961628/v1

License: (c) (1) This work is licensed under a Creative Commons Attribution 4.0 International License. Read Full License 


\section{Abstract}

\section{Background}

Idiopathic scoliosis (IS) is the most common structural scoliosis, which seriously affects not only patient's physical and mental health but also quality of patient's life. Abnormal osteogenic differentiation of bone marrow mesenchymal stem cells (BMSCs) is one of the causes of IS. However, the regulation mechanism of osteogenic differentiation of BMSCs in patients with IS remains to be further studied.

\section{Methods}

Serum samples of 135 patients with IS were collected, and the expression of miRNA were detected by RTqPCR. BMSCs from patients with IS were collected and the expression of miR-192-5p in BMSCs from IS patients and normal BMSCs was detected by RT-qPCR. Double luciferase reporter genes assay was used to verify the targeting relationship between miR-192-5p and RSPO1. The levels of RSP01, osteogenic related proteins (OC, OPN and RUNX2) and Wnt/ $\beta$-catenin signaling pathway related proteins (WNT3A and $\beta$-catenin) were detected by Western blotting. Alkaline phosphatase staining and alizarin red staining were used to evaluate the osteogenesis of BMSCs.

Results

miR-192-5p was significantly up-regulated in serum and BMSCs of patients with IS. Alkaline phosphatase staining and alizarin red staining showed that miR-192-5p inhibitor promoted the osteogenic differentiation of BMSCs from IS patients. miR-192-5p targeted down-regulated the expression of RSP01 in BMSCs from IS patients. In addition, overexpression of RSP01 activated Wnt/ $\beta$-catenin signaling pathway in BMSCs from IS patients. Furthermore, miR-192-5p/RSP01 axis regulated levels of osteogenic related proteins (OC, OPN and RUNX2) in BMSCs from IS patients through Wnt/ $\beta$-catenin signaling pathway, and affected the osteogenic differentiation of BMSCs.

\section{Conclusion}

miR-192-5p, which was highly expressed in patients with IS, inhibited Wnt/ $\beta$-catenin signaling pathway by down-regulating RSPO1 protein and then reduced the osteogenic differentiation ability of BMSCs.

\section{Background}

Scoliosis refers to the deviation in the normal vertical spine, which is the most common deformity of the spine.[1] As one pattern of scoliosis, idiopathic scoliosis (IS) seriously affects not only patient's physical and mental health but also quality of patient's life.[2] According to the research on IS, the available causes included genetic factors, hormonal factors, bone and connective tissue anomalies.[3] Focus on the role of bone loss plays in cause of IS, there has been reported that the mechanism and causes of bone loss in adolescent IS might be decrease osteogenic differentiation of mesenchymal stem cells.[4] 
Emerging evidence showed that microRNAs (miRNAs) are deregulated in scoliosis.[5] Importantly, there was also a study found that the differential expression of miRNAs in Bone marrow mesenchymal stem cells (BMSCs) from adolescent IS patients might provide a deep insight into the cause of adolescent IS. [6] Nevertheless, the mechanism that miRNAs regulate the osteogenic differentiation of BMSCs involve in the pathogenesis of IS is still not clear.

R-spondin (RSPO) family belong to a superfamily of thrombospondin type 1 repeat-containing proteins, and contain four secreted proteins, RSP01-4. RSPO secreted cysteine-rich glycoproteins and play an important role in maintenance of stem cells.[7] Moreover, RSPO together with WNT proteins enhance the activity of canonical Wnt/ $\beta$-catenin signaling pathway.[8] Interestingly, activation of Wnt/ $\beta$-catenin signaling pathway in BMSCs promoted osteogenic differentiation and reversed bone loss.[9] However, there was no study on the relationship between RSPO and IS.

In this study, the differential expression of miRNAs in serum and BMSCs samples form IS patients was measured and the underlying mechanisms of miR-192-5p in osteogenic differentiation of BMSCs in IS was explored to provide a new target for the prevention and treatment of IS.

\section{Methods}

\section{Serum and BMSCs samples}

From January 2016 to December 2019, the serum samples of 118 normal person and 135 IS patients were collected at First Affiliated Hospital of Kunming Medical University. And BMSCs samples from IS patients without other disease related to bone were collected as well. All patients have signed the informed consent. All experiments in this study approved by Ethics committee of First Affiliated Hospital of Kunming Medical University with the number (2016) Ethical approval L NO. 48 and conformed to the World Medical Association Declaration of Helsinki.

\section{Cell culture and transfection}

BMSCs collected from IS patients and normal BMSCs (\#FY200001, Fuyuanbio, Shanghai, China) purchased from Fuyuan (Shanghai) Biotechnology Co., Ltd were cultured in complete medium with 10\% fetal bovine serum (\#gibco-10099-141, Gibco, Australia), $100 \mathrm{U} / \mathrm{ml}$ penicillin (\#K0031, Baomanbio, Shanghai, China) and $100 \mu \mathrm{g} / \mathrm{ml}$ streptomycin (\#K0107, Baomanbio) under an atmosphere of $37^{\circ} \mathrm{C}$ and $5 \% \mathrm{CO}_{2}$. miR-192-5p mimics, miR-192-5p inhibitor, pcDNA-RSP01 and si-RSP01 were designed and produced by Guangzhou Ruibo Biotechnology Co., Ltd (China). Cell transfections were carried on by using Lipofectamine2000 (\#11668-019, Invitrogen, Carlsbad, USA) according to manufacturer's instructions.

\section{RT-qPCR}

Extraction of total RNA enriched with miRNA was performed by using TRIzol reagent (\#B0201, HaiGene, Harbin, China). And then reversed into cDNA with TruScript Reverse Transcriptase Kit (\#54440-E, Norgen 
Biotek, CA). After that, RT-qPCR was carried on with a total reaction volume of $20 \mu \mathrm{L}$ which containing 2 $\mu \mathrm{L}$ of cDNA by using TB Green Premix Ex Taq II (\#RR820A; TAKARA, Japan). The primers were as follows: miR-192-5p F: 5'-GGACTTTCTTCATTCACACCG-3' and R 5'-GACCACTGAGGTTAGAGCCA-3'; RSP01 F: 5'TGTGAAATGAGCGAGTGGTCC-3' and R 5'-TCTCCCAGATGCTCCAGTTCT-3'; U6 F: 5'-

CTCGCTTCGGCAGCACA-3' and R 5'-AACGCTTCACGAATTTGCGT-3'; GAPDH F: 5'-

TCGACAGTCAGCCGCATCTTCTTT-3' and R 5'-GCCCAATACGACCAAATCCGTTGA-3'. U6 or GAPDH was used as an internal standard. The relative expression of miRNA and mRNA was calculated based on the $2^{-\Delta \Delta \mathrm{Ct}}$ method.

\section{Western blotting}

Total proteins were extracted by using RIPA lysis buffer (\#20101ES60, Yeasen, Shanghai, China) and the concentration of protein was detected by using BCA protein quantification kit (\#20201ES76, Yeasen). Proteins were separated and transferred using 10\% SDS-PAGE and then transferred to PVDF membranes. The PVDF membranes were blocked in $5 \%$ skimmed milk at room temperature for $2 \mathrm{~h}$. And then incubated with primary antibodies at $4{ }^{\circ} \mathrm{C}$ overnight. Primary antibodies were CD44 (1:1000, \#bs-0521R, Bioss, Beijing, China), CD90 (1:1000, \#bs-0778R, Bioss), CD34 (1:1000, \#bs-8996R, Bioss), CD 45 (1:1000, \#bs10599R, Bioss), anti-RSP01 (1:1000, \#PA520749, Thermo Scientific, USA), anti-OC (1:1000, \#YJ81371, Yiji, Shanghai, China), anti-OPN (1:1000, \#ZIKER-0019R, ZIKER, Shenzhen, China), anti-RUNX2 (1:1000, \#ZIKER-1134R, ZIKER), anti-WNT3A (1:1000, \#NY-231688M, Anyan, Nanjing, China), anti- $\beta$-catenin (1:1000, \#CYm-33071M, Caiyou, Shanghai, China) and anti-GAPDH (1:1000, \#BM1985, BOSTER, Wuhan, China). In the next day, the membranes were incubated at room temperature with secondary antibody (1:1000, \#zk2597, ZIKER) for $1 \mathrm{~h}$. The enhanced ECL chemiluminescence detection kit (\#HR0340-DXC, Biomart, Beijing, China) was used to detect blot. GAPDH was used as an internal standard.

\section{Alkaline phosphatase (ALP) and Alizarin red S (ARS) staining}

After washed the cells with PBS for three times, fixed them with 4\% paraformaldehyde (\#36314ES60, Yeasen) at $37^{\circ} \mathrm{C}$ for $30 \mathrm{~min}$. Subsequently, according to the manufacturer's instructions ALP staining was performed by using Alkaline Phosphatase Stain Kit (\#40749ES60, Yeasen) and ARS staining was performed by using Alizarin Red S Staining Kit (\#C-8309, Caiyou). Then observed and photographed the cells under a microscope.

\section{Double luciferase reporter genes assay}

The sequence of the RSP01 wild type (RSP01-WT) or RSP01 mutant type (RSP01-MUT) and miR-192-5p mimics were co-transfected into 293T cells (\#HZ-H321, ATCC, USA) by using Lipofectamine2000. After transfection for $48 \mathrm{~h}$, the luciferase activity was measured by dual luciferase reporter assay system.

\section{Statistical analysis}


All the data in this study were expressed as means \pm standard deviation and calculated by SPSS 19.0. The difference between two groups was assessed by Student's $t$ test and the difference in multiple groups was analyzed by one-way ANOVA. The differences of $p<0.05$ was considered statistically significant.

\section{Results}

\section{Expression of miR-192-5p in IS patients' serum and BMSCs}

It has been reported that miRNAs participate in the process of IS. According to the heat map results (Fig. $1 a)$, this study focused on the role of miR-192-5p played in IS. RT-qPCR results showed that the expression of miR-192-5p was significant higher in the serum samples of IS patients than normal people (Fig. 1b). In order to explored the function of miR-192-5p in BMSCs of IS, the BMSCs were obtained from IS patients, and western blotting showed that the BMSCs marker CD44 and CD90 were positive, CD34 and CD45 were negative (Fig. 1C). In addition, compared with the normal BMSCs, there was increased miR-192-5p expression in BMSCs from IS patients (Fig. 1d). Hence, the abnormal high expression of miR-192-5p in serum and BMSCs from IS patients might contribute to occurrence and deterioration of IS.

\section{Function of miR-192-5p in BMSCs from IS patient}

Based on the above results, miR-192-5p expressed abnormality greatly in BMSCs from IS patient, which might play an important role in osteogenic differentiation of BMSCs. In order to explored the function of miR-192-5p in BMSCs from IS patient, miR-192-5p inhibitor was transfected into the cells at first (Fig. 2a). Western blotting results showed that the expression of osteogenic related proteins (OC, OPN and RUNX2) increased in miR-192-5p inhibitor group compared with the NC group (Fig. 2b). Alkaline phosphatase staining (Fig. 2c) and alizarin red staining (Fig. 2d) also revealed that knockdown of miR-192-5p enhanced the osteogenic differentiation of BMSCs in IS. Therefore, miR-192-5p effected on the osteogenic differentiation of BMSCs in IS patient.

\section{Relationship of miR-192-5p, RSP01 and Wnt/ $\beta$-catenin signaling pathway}

miRNAs play some roles in cells through interact with target gene. The binding sites between miR-192-5p and RSP01 were showed as Fig. 3a. Moreover, double luciferase reporter gene results showed that compared with the NC group, the luciferase activity of RSP01-WT significantly reduced in miR-192-5p mimics group, and the luciferase activity of RSP01-MUT showed no significantly differences between NC and miR-192-5p mimics group (Fig. 3b). In addition, transfected miR-192-5p mimics in the BMSCs from IS patient, after testify the transfection efficiency (Fig. 3c), Western blotting was used to detect the expression of RSP01 protein, the results showed that overexpression of miR-192-5p downregulated RSPO1 (Fig. 3d). Besides, RSPO1 is a regulator of Wnt/ $\beta$-catenin signaling pathway. The RSP01 was overexpressed or knock downed by pcDNA-RSPO1 or si-RSP01 in the BMSCs from IS patient (Fig. 3e), Western blotting results showed that the expression of WNT3A and $\beta$-catenin increased 
with the increase of RSP01, and the expression of WNT3A and $\beta$-catenin decreased with the decrease of RSP01 (Fig. 3f). Thus, miR-192-5p inhibited Wnt/ $\beta$-catenin signaling pathway through down-regulated the expression of RSP01.

\section{miR-192-5p effected on osteogenic differentiation of BMSCs in IS by regulating Wnt/ $\beta$-catenin signaling pathway via RSP01}

Furthermore, the mechanism that miR-192-5p effected on osteogenic differentiation of BMSCs in IS by regulating Wnt/ $\beta$-catenin signaling pathway via RSP01 was studied. FH535 is inhibitor of Wnt/ $\beta$-catenin signaling pathway. Western blotting results showed that compared with the NC group, the expression of Wnt/ $\beta$-catenin signaling pathway related proteins (WNT3A and $\beta$-catenin) and osteogenic related proteins (OC, OPN and RUNX2) markedly raised in the OE-RSPO1 group, but there was no significant difference between the OE-RSPO1+miR-192-5p mimics group and the NC group; besides, the expression of WNT3A, $\beta$-catenin, OC, OPN and RUNX2 remarkable reduced in the OE-RSP01+FH535 group compared with the OE-RSPO1 group (Fig. 4a). Alkaline phosphatase staining (Fig. 4b) and alizarin red staining (Fig. 4c) results showed that miR-192-5p mimics or FH535 reversed the promotion of osteogenic differentiation induced by OE-RSPO1 in BMSCs. Therefore, miR-192-5p restrained osteogenic differentiation of BMSCs in IS by down-regulated RSPO1 and inhibited Wnt/ $\beta$-catenin signaling pathway.

\section{Discussion}

IS identify when scoliosis patients have no other underlying disease. For those IS patients who have not yet reached skeletal maturity, the treatment is often prophylactic. For adult IS patients, the treatment is most frequently because of symptoms, such as back pain, deformities, respiratory and cardiology problems.[10] [11] Due to a currently recognizable cause of IS has not been found, it is urgent to explore the underlying mechanism of IS pathogeny.

Bone loss is one of the most important causes of IS.[12] With the research on miRNAs, there was a study reported that miRNAs can be potentially used as a clinical tool for molecular IS diagnosis, and this study also evidence for the deregulated miRNAs that participate in osteoblast or osteoclast differentiation mechanisms in IS.[13] For example, Bcl2-targeted miR-15a was downregulated in IS patients, which related to chondrocyte proliferation and participate in the development and progression of IS.[14] Besides, recent report confirmed that aberrant miR-145-5p impaired osteocyte function in adolescent IS.

[15] Osteogenic differentiation of BMSCs is closely related to bone formation.[16] In this study, we found that miR-192-5p highly expressed in not only the serum but also BMSCs of IS patients. Moreover, knockdown of miR-192-5p BMSCs from IS patients inhibited osteogenic differentiation. The results elucidated that miR-192-5p might involve in IS through regulating osteogenic differentiation of BMSCs.

miRNAs contribute to differentiation of stem cells by regulating target genes and important cellular pathways. Based on the prediction of starbase, RSP01 as a target gene of miR-192-5p participated in apoptosis of BMSCs.[17] Additionally, RSPO1 may interact with Wnt/ $\beta$-catenin signaling pathway to induce differentiation in osteoblasts.[18] Our study results illuminated that overexpression of miR-192-5p 
or inhibition of Wnt/ $\beta$-catenin signaling pathway in BMSCs reversed the promotion of osteogenic differentiation induced by overexpression of RSP01.

About the osteogenic differentiation related proteins OC, OPN and RUNX2 in IS, a new study claimed that the level of OC in the severe adolescent IS patients was significantly lower.[19] Besides, OPN was at a low level in adolescent IS bone tissues.[15] And RNA and protein analyses revealed lower RUNX2 levels in adolescent IS.[20] The results in this study showed that inhibition of miR-192-5p or overexpression of RSPO1 increased the level of OC, OPN and RUNX2 in BMSCs from IS patients, this results emphasized that miR-192-5p and RSP01 might be exploited as a potential target for IS therapy.

\section{Conclusion}

In conclusion, miR-192-5p, which was highly expressed in patients with IS, inhibited Wnt/ $\beta$-catenin signaling pathway by down-regulating RSPO1 protein and then reduced the osteogenic differentiation ability of BMSCs.

\section{Abbreviations}

BMSCs: Bone marrow mesenchymal stem cells; IS: idiopathic scoliosis; ALP: Alkaline phosphatase; RTqPCR: Real-time quantitative polymerase chain reaction; WNT3A: WNT family member 3a; OC: Osteocalcin; OPN: Osteopontin; RUNX2: Runt-related gene 2; RSP01: R-spondin 1; miRNAs: MicroRNAs; NC: Negative control; OE: Overexpressed; WT: Wild type; MUT: Mutant type; siRNA: Small interfering RNA

\section{Declarations}

\section{Acknowledgements}

Not applicable.

\section{Authors' contributions}

TD, YFY and JX designed this study. YFY, JX, QXS and YRW performed the experiment. QZL and ZRM analyzed the results. All authors read and approved the final manuscript.

\section{Funding}

Not applicable.

\section{Availability of data and materials}

All the data in this study are available from the corresponding author on reasonable request.

\section{Ethics approval and consent to participate}


All experiments in this study approved by Ethics committee of First Affiliated Hospital of Kunming Medical University with the number (2016) Ethical approval L NO. 48 and conformed to the World Medical Association Declaration of Helsinki.

\section{Consent for publication}

Not applicable

\section{Competing interests}

The authors declare that they have no competing interests.

\section{Author details}

${ }^{1}$ Department of Rehabilitation Medicine, First Affiliated Hospital of Kunming Medical University, No 295 Xichang Road, Xishan District, Kunming, China. ${ }^{2}$ Department of traditional Chinese medicine, First Affiliated Hospital of Kunming Medical University, No 295 Xichang Road, Xishan District, Kunming, China

\section{References}

1. Shakil H, Iqbal ZA, Al-Ghadir AH: Scoliosis: review of types of curves, etiological theories and conservative treatment. Journal of back and musculoskeletal rehabilitation 2014, 27(2):111-115.

2. Rodrigues JB, Saleme NA, Batista JL, Jr., Cardoso IM, Jacob C, Jr.: Quality of life in patients submitted to surgical treatment of idiopathic scoliosis. Acta ortopedica brasileira 2015, 23(6):287-289.

3. Yaman O, Dalbayrak S: Idiopathic scoliosis. Turkish neurosurgery 2014, 24(5):646-657.

4. Park WW, Suh KT, Kim JI, Kim SJ, Lee JS: Decreased osteogenic differentiation of mesenchymal stem cells and reduced bone mineral density in patients with adolescent idiopathic scoliosis. European spine journal : official publication of the European Spine Society, the European Spinal Deformity Society, and the European Section of the Cervical Spine Research Society 2009, 18(12):1920-1926.

5. Li Z, Li X, Shen J, Zhang L, Chan MTV, Wu WKK: Emerging roles of non-coding RNAs in scoliosis. Cell proliferation 2020, 53(2):e12736.

6. Hui S, Yang Y, Li J, Li N, Xu P, Li H, Zhang Y, Wang S, Lin G, Li S et al: Differential miRNAs profile and bioinformatics analyses in bone marrow mesenchymal stem cells from adolescent idiopathic scoliosis patients. The spine journal : official journal of the North American Spine Society 2019, 19(9):1584-1596.

7. Li Z, Liu S, Lou J, Mulholland M, Zhang W: LGR4 protects hepatocytes from injury in mouse. American journal of physiology Gastrointestinal and liver physiology 2019, 316(1):G123-g131. 
8. Raslan AA, Yoon JK: R-spondins: Multi-mode WNT signaling regulators in adult stem cells. The international journal of biochemistry \& cell biology 2019, 106:26-34.

9. Hong G, He X, Shen Y, Chen X, Yang F, Yang P, Pang F, Han X, He W, Wei Q: Chrysosplenetin promotes osteoblastogenesis of bone marrow stromal cells via Wnt/ $\beta$-catenin pathway and enhances osteogenesis in estrogen deficiency-induced bone loss. Stem cell research \& therapy 2019, 10(1):277.

10. Angevine PD, Deutsch H: Idiopathic scoliosis. Neurosurgery 2008, 63(3 Suppl):86-93.

11. Jandrić S: [Idiopathic scoliosis]. Medicinski pregled 2012, 65(1-2):35-40.

12. Diarbakerli E, Savvides P, Wihlborg A, Abbott A, Bergström I, Gerdhem P: Bone health in adolescents with idiopathic scoliosis. The bone \& joint journal 2020, 102-b(2):268-272.

13. García-Giménez JL, Rubio-Belmar PA, Peiró-Chova L, Hervás D, González-Rodríguez D, IbañezCabellos JS, Bas-Hermida P, Mena-Mollá S, García-López EM, Pallardó FV et al: Circulating miRNAs as diagnostic biomarkers for adolescent idiopathic scoliosis. Scientific reports 2018, 8(1):2646.

14. Li J, Yang G, Liu S, Wang L, Liang Z, Zhang H: Suv39h1 promotes facet joint chondrocyte proliferation by targeting miR-15a/Bcl2 in idiopathic scoliosis patients. Clinical epigenetics 2019, 11(1):107.

15. Zhang J, Chen H, Leung RKK, Choy KW, Lam TP, Ng BKW, Qiu Y, Feng JQ, Cheng JCY, Lee WYW: Aberrant miR-145-5p/ $\beta$-catenin signal impairs osteocyte function in adolescent idiopathic scoliosis. FASEB journal : official publication of the Federation of American Societies for Experimental Biology 2018:fj201800281.

16. Dong CL, Liu XH, Wu L: [Research and development of osteogenic differentiation of bone marrow mesenchymal stem cells]. Zhongguo gu shang = China journal of orthopaedics and traumatology 2019, 32(3):288-292.

17. Cheng XX, Yang QY, Qi YL, Liu ZZ, Liu D, He S, Yang LH, Xie J: Apoptosis of mesenchymal stem cells is regulated by Rspo1 via the Wnt/ $\beta$-catenin signaling pathway. Chronic diseases and translational medicine 2019, 5(1):53-63.

18. Sharma AR, Choi BS, Park JM, Lee DH, Lee JE, Kim HS, Yoon JK, Song DK, Nam JS, Lee SS: Rspo 1 promotes osteoblast differentiation via Wnt signaling pathway. Indian journal of biochemistry \& biophysics 2013, 50(1):19-25.

19. Matusik E, Durmala J, Olszanecka-Glinianowicz M, Chudek J, Matusik P: Association between Bone Turnover Markers, Leptin, and Nutritional Status in Girls with Adolescent Idiopathic Scoliosis (AIS). Nutrients 2020, 12(9). 
20. Xiao L, Zhang H, Wang Y, Li J, Yang G, Wang L, Liang Z: Dysregulation of the ghrelin/RANKL/OPG pathway in bone mass is related to AIS osteopenia. Bone 2020, 134:115291.

\section{Figures}

A

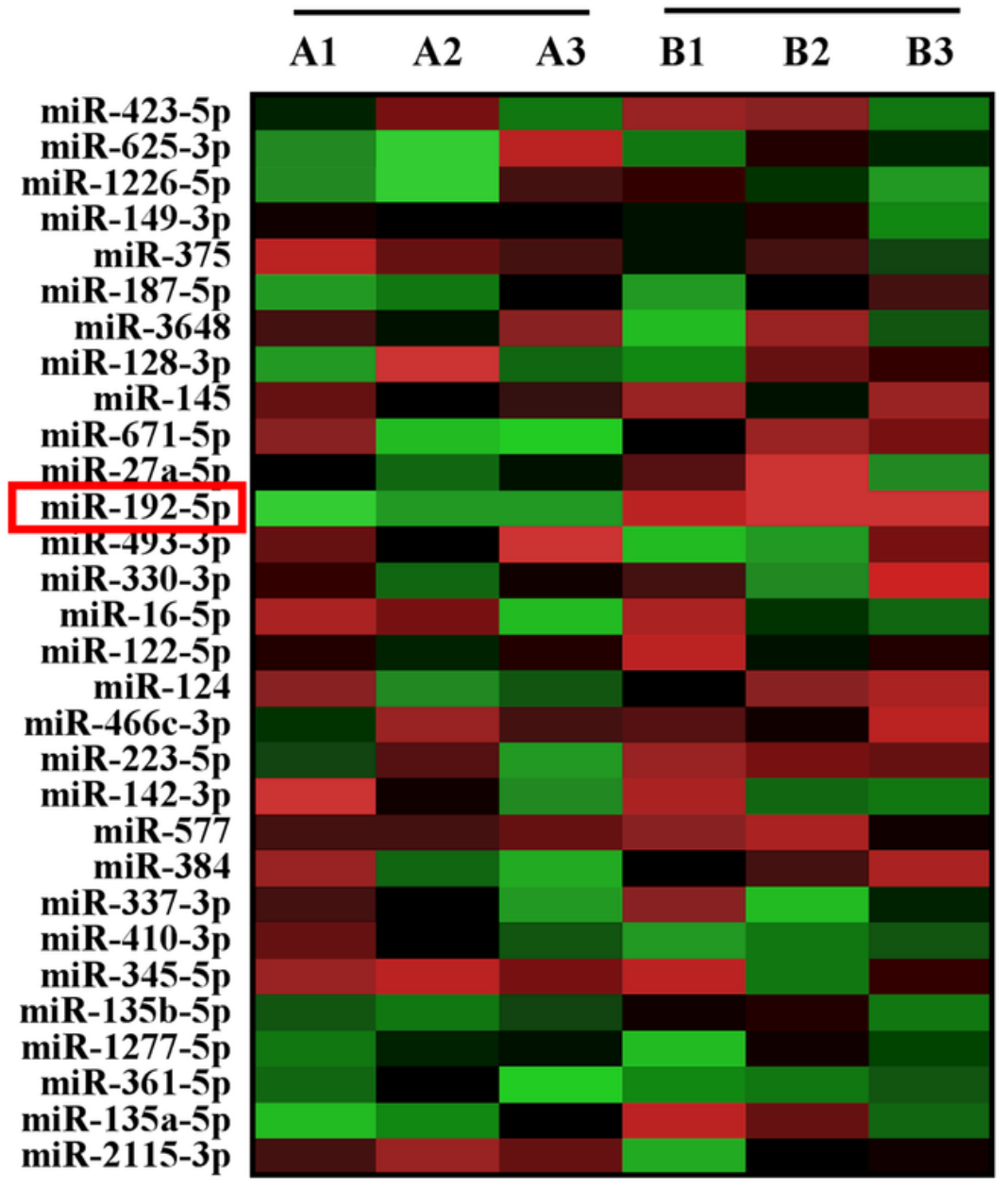

B
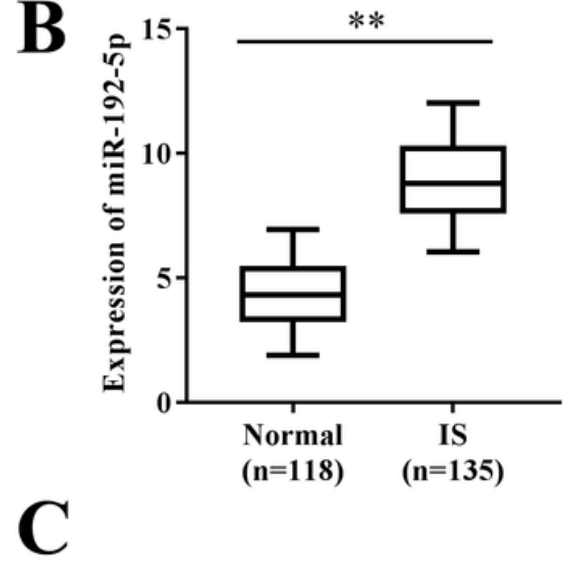

$-2$

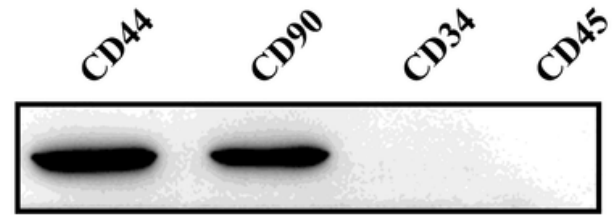

$-3$
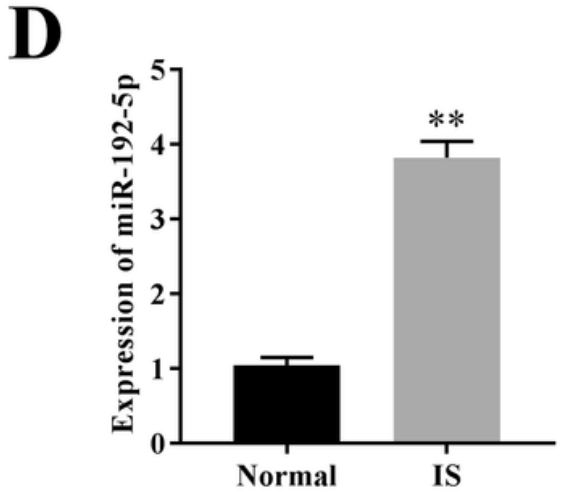

Figure 1

Expression of miR-192-5p in IS patients' serum and BMSCs. a Heat map of miRNAs expression in serum from normal person (A1-3) and IS patients (B1-3) was examined by RT-qPCR. b Expression of miR-192-5p in serum from normal person and IS patients was measured by RT-qPCR. c BMSCs marker (CD44, CD90, CD34 and CD45) of BMSCs from IS patients was detected by flow cytometry. $d$ Expression of miR-192-5p in BMSCs from normal person and IS patients was measured by RT-qPCR. ${ }^{*} \mathrm{P}<0.01$ vs Normal group 


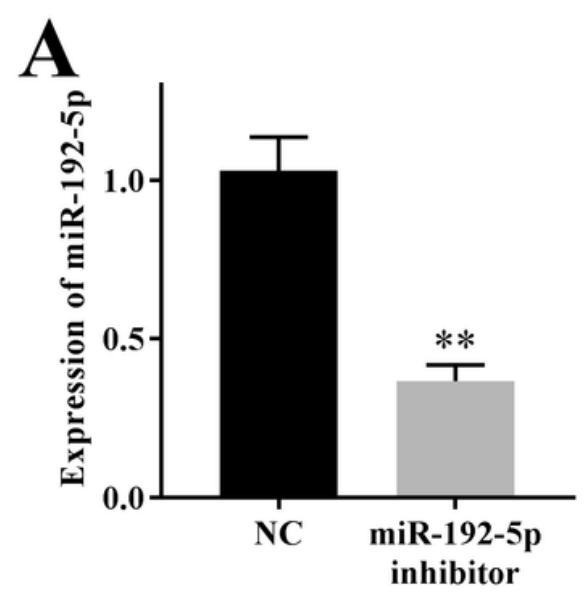

C ALP staining
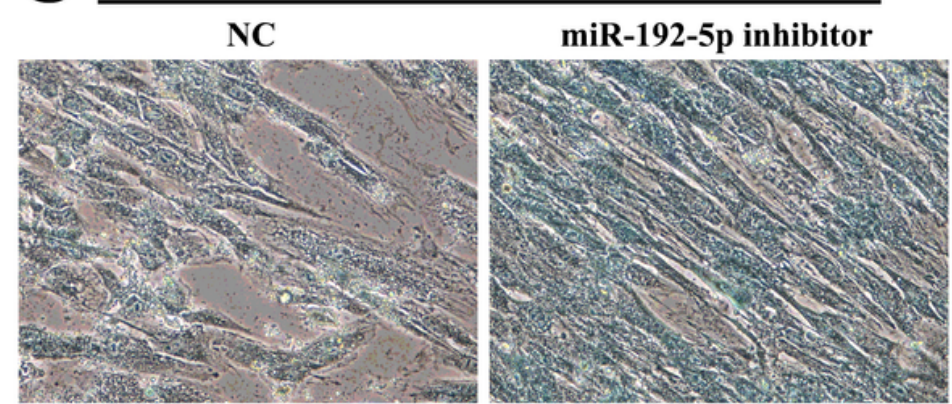
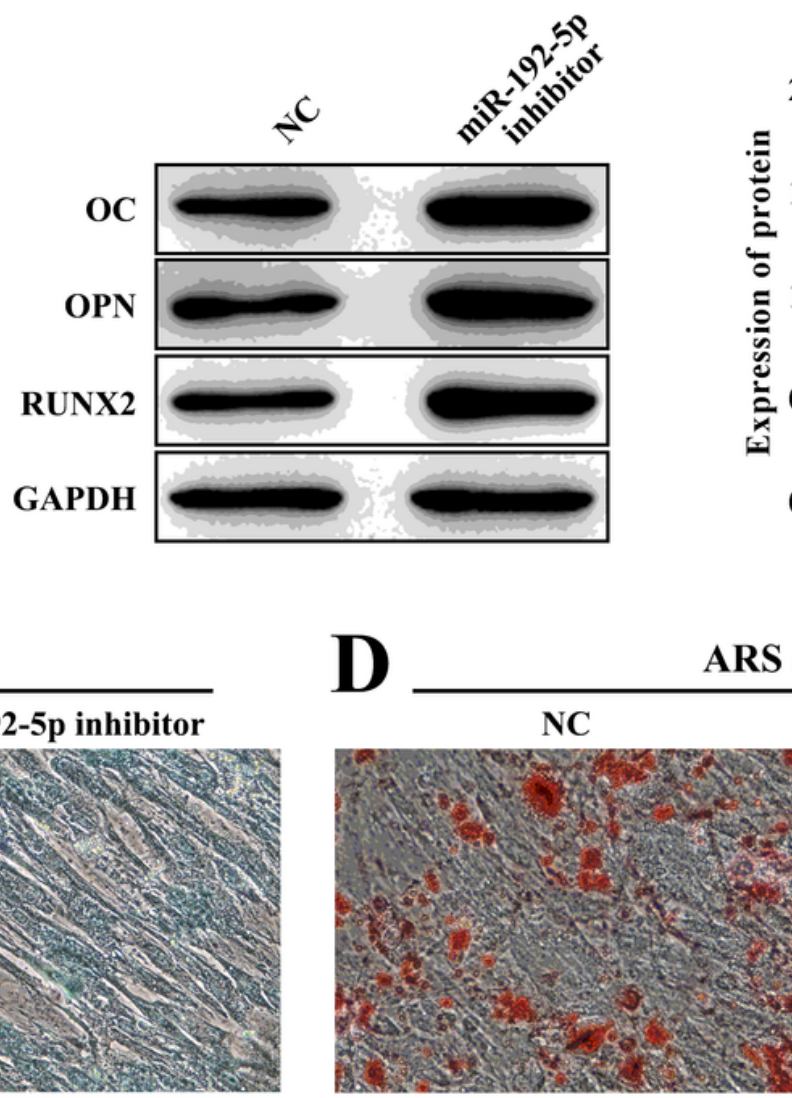

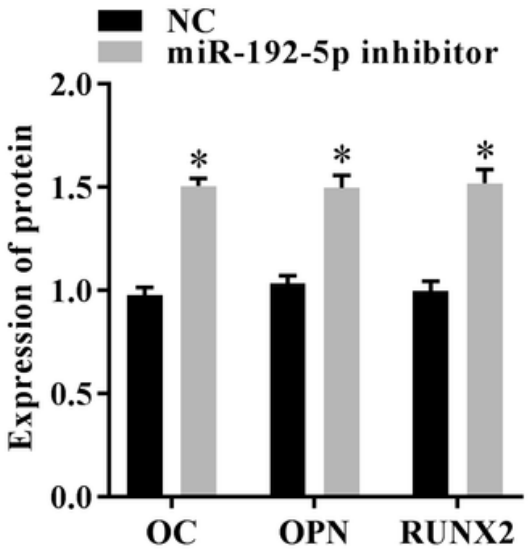

ARS staining

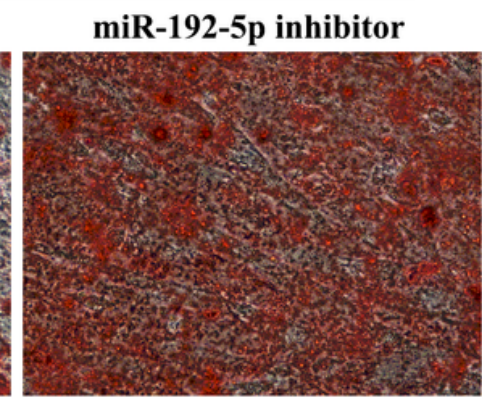

Figure 2

Function of miR-192-5p in BMSCs from IS patient. a Transfection efficiency of miR-192-5p inhibitor in BMSCs from IS patients was examined by RT-qPCR. b Expression of osteogenic related proteins (OC, OPN and RUNX2) in BMSCs from IS patients was measured by Western blotting. c Alkaline phosphatase (ALP) staining results of BMSCs from IS patients. $d$ Alizarin red $S$ (ARS) staining results of BMSCs from IS patients. ${ }^{* *} \mathrm{P}<0.01$ vs NC group 

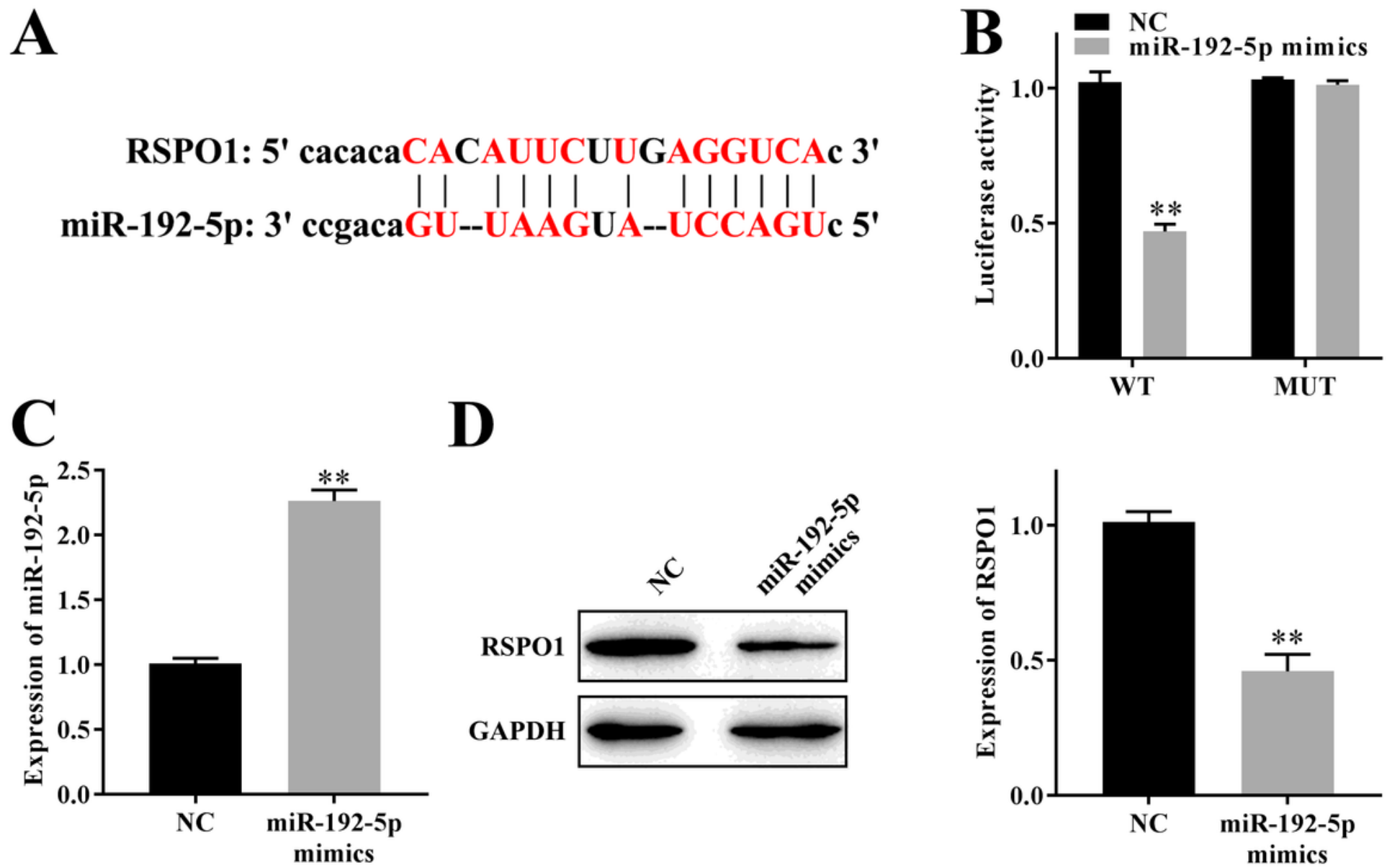

D

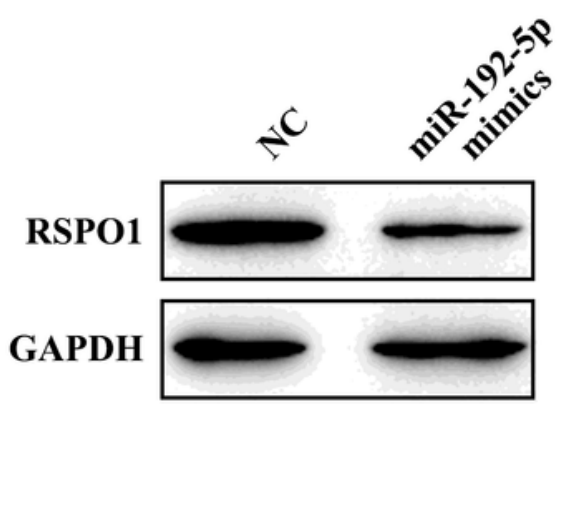

$\mathbf{E}$

$\mathbf{F}$
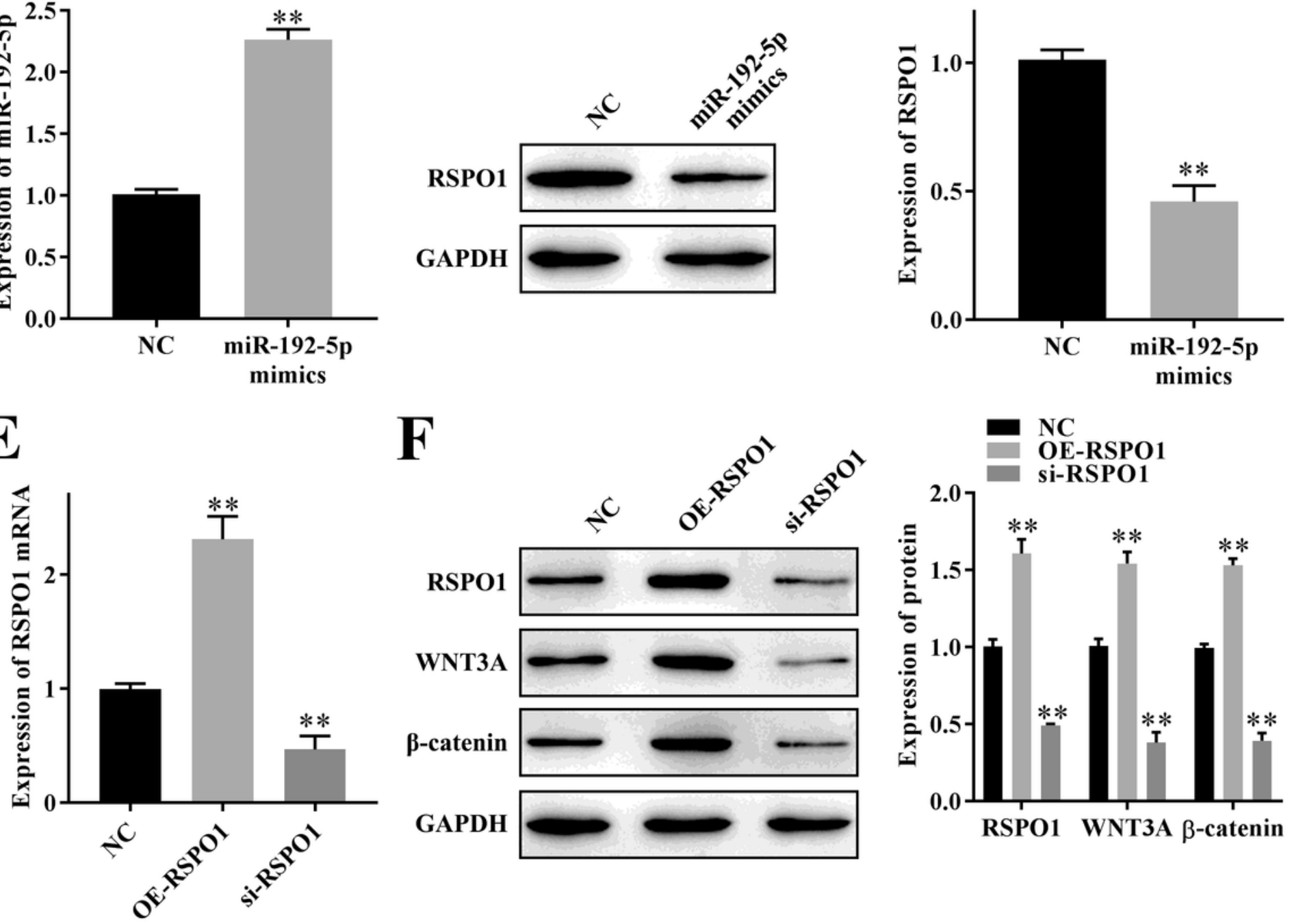

Figure 3

Relationship of miR-192-5p, RSPO1 and Wnt/ $\beta$-catenin signaling pathway. a The binding sites between miR-192-5p and RSP01. b Target relationship between miR-192-5p and RSP01 was verified by Double luciferase reporter genes assay. $c$ Transfection efficiency of miR-192-5p mimics in BMSCs from IS patients was examined by RT-qPCR. $d$ Expression of RSP01 in BMSCs from IS patients was measured by Western blotting. e Transfection efficiency of pcDNA-RSP01 or si-RSP01 in BMSCs from IS patients was 
examined by RT-qPCR. $f$ Expression of RSP01 and Wnt/ $\beta$-catenin signaling pathway related proteins (WNT3A and $\beta$-catenin) in BMSCs from IS patients was measured by Western blotting. ${ }^{* *} \mathrm{P}<0.01$ vs NC group

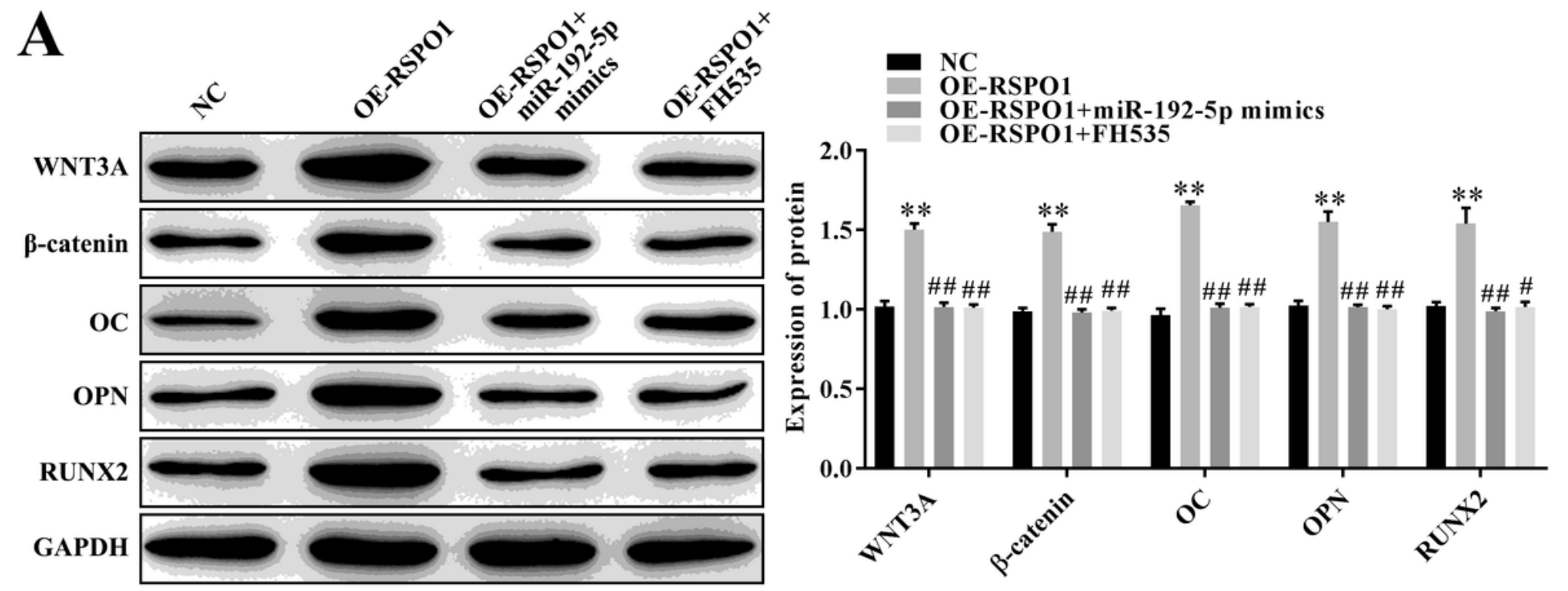

B

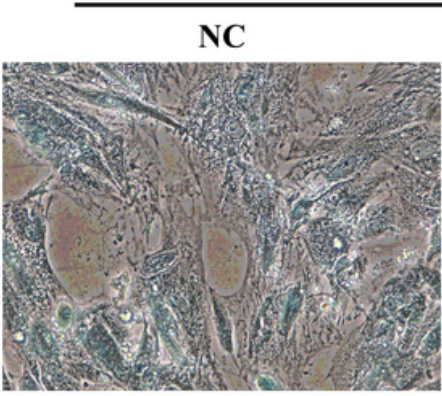

ALP staining
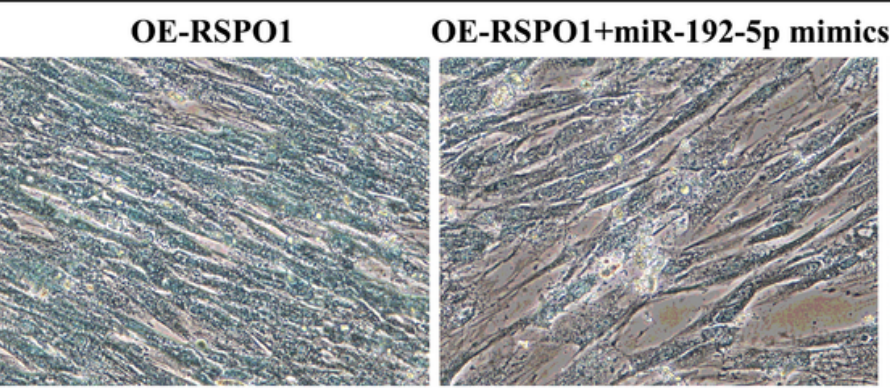

OE-RSPO1+FH535

ARS staining

C
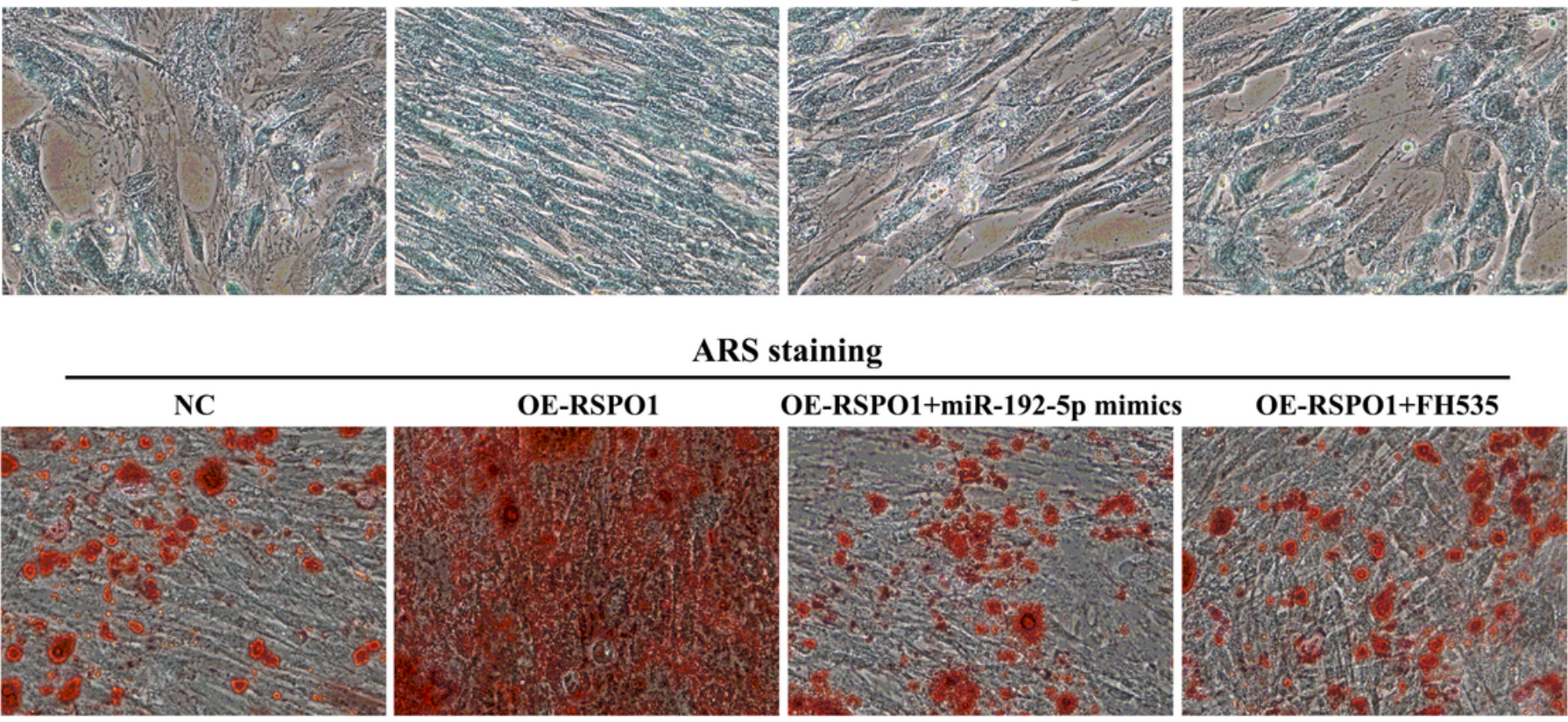

\section{Figure 4}

miR-192-5p effected on osteogenic differentiation of BMSCs in IS by regulating Wnt/ $\beta$-catenin signaling pathway via RSP01. a Expression of osteogenic related proteins (OC, OPN and RUNX2) and Wnt/ $\beta$ catenin signaling pathway related proteins (WNT3A and $\beta$-catenin) in BMSCs from IS patients was measured by Western blotting. b Alkaline phosphatase (ALP) staining results of BMSCs from IS patients. c Alizarin red S (ARS) staining results of BMSCs from IS patients. ${ }^{\star}{ }^{\mathrm{P}}<0.01$ vs NC group, \#\#P<0.01 vs 0 RSP01 group 


\section{Supplementary Files}

This is a list of supplementary files associated with this preprint. Click to download.

- EthicscommitteePAGE1.jpg

- EthicscommitteePAGE2.jpg 